\title{
Household Food Insecurity, Coping Strategies and Child Dietary Diversity (24-59 months) in Ibadan, Nigeria
}

Authors: Folake Olukemi Samuel ${ }^{1}$; Ifeoluwa Omolara Otitoju ${ }^{1}$ and Akinkunmi Paul Okekunle ${ }^{2,3}$

Authors' institutional affiliations:

${ }^{1}$ Department of Human Nutrition, College of Medicine, University of Ibadan. Nigeria.

${ }^{2}$ Department of Epidemiology and Medical Statistics, College of Medicine, University of Ibadan, Ibadan Nigeria.

${ }^{3}$ The Postgraduate College, University of Ibadan, 200284 Ibadan Nigeria

Corresponding author: Ifeoluwa Omolara Otitoju (otitojuifeoluwa@gmail.com) 


\section{Abstract}

Background and Objective: The importance of optimal nutrition should not be underestimated in population well-being and national development. The aim of this study is to assess the relationship between household food insecurity (HFI) and child dietary diversity (CDD) in motherchild (24-59 months) pairs in an urban population.

Method: HFI and CDD were assessed using primary data collected from a sample of 360 apparently healthy mother-child pairs from three local government wards in Ibadan, Nigeria. HFI was categorized as 'food secure' and 'food insecure' and the CDD score (CDDS) was classified as low $(<4)$ and high $(\geq 4)$. Multiple logistic regression models were used to assess the association between HFI and CDD at $P<0.05$.

Results: $90.3 \%$ of households were food insecure and $30.0 \%$ of children had a low CDDS. The proportion of children with low CDD was significantly higher $(\mathrm{P}=0.012)$ among food-insecure households (32.0\%) compared to children from food-secure households $(11.4 \%)$. Similarly, the multivariable-adjusted odds ratio of high CDD given HFI was 0.266 (95\% confidence interval 0.091, 0.783), $P=0.016$.

Conclusion: HFI was associated with the risk of low CDD and children from food-insecure households are about four times less likely to present high CDDS. It is vital to affirm the significance of HFI in the risk of low CDDS using a large multi-ethnic longitudinal cohort taking into cognizance relevant confounders.

Keywords: Household food insecurity, Dietary diversity, Coping strategies, Nutritional status, Children 


\section{INTRODUCTION}

The interconnection of social, economic, and biophysical systems to meet people's nutritional requirements is a strong determining factor for food insecurity (FAO 2018). According to FAO, food security is "when all people, at all times, have physical, social and economic access to sufficient, safe and nutritious food to meet their dietary needs and food preferences for an active and healthy life.” (FAO 2018). Food security on a family level can be determined in two main ways: food production and food purchase that requires adequate resources or income (Ajao et al. 2010). The importance of optimal nutrition should not be underestimated in population well-being and national development. On the other hand, economic growth stands as the crux in the fight against hunger and poverty, especially in the state of sporadic population growth (FAO 2015). Household food insecurity (HFI) is a useful proxy for understanding public health nutrition situations and crucial for meeting international nutrition targets. In addition, it is a suitable index for monitoring and achieving the sustainable development goals (SDG) 2 and 3. However, evidence-based primary data on the HFI status of the population in low resource settings is usually lacking, particularly in times of economic downturn. HFI is an underlying determinant of child under-nutrition and consequently informs cognitive capacity and economic productivity in adulthood (Black et al. 2008). In Nigeria as in other settings, HFI is usually accompanied by coping strategies (CS), however, there are few local studies reporting these, making it challenging to design public health intervention strategies that are vital for the management of HFI in Nigeria. Coping strategies imply all actions adopted by individuals and households to limit their expenses or gain extra income in order to pay for basic necessities and not be relegated too far behind society's level of welfare (Snel and Staring 2001). Understanding household level CS is vital to discerning adaptive strategies in the HFI situation (Farzana et al. 2017).

Dietary diversity is a measure of individual/household food access to provide an all-inclusive overview of the food and nutrition security status in a community (Kennedy, Ballard, and Dop 2011). However, whether HFI (particularly in economically-tense situations) is associated with child dietary diversity (CDD) is not clearly understood. It is a suitable proxy that provides insights into the consumption of a wide variety of foods represented in a child's diet (Ogundari 2013, Pera, Katz, and Bentley 2019). A child's nutritional status is directly affected by dietary consumption and the absence of infectious diseases that affect child growth (Black et al. 2013). The household dietary diversity score (HDDS) is intended to depict at a glance the economic ability of a household to have a variety of foods while individual dietary diversity scores are intended to reflect nutrient adequacy (Kennedy, Ballard, and Dop 2011). Dietary diversity as a measure of household food access and food consumption can be combined with other food-related information to in order to bring about an all-inclusive picture of the food and nutrition security status in a community (Kennedy, Ballard, and Dop 2011). Dietary diversity is the consumption of a wide variety of foods represented in an individual's diet that cuts across nutritionally distinct food groups developed by the World Food Program (WFP) (Ogundari 2013, Pera, Katz, and Bentley 2019). It has been associated with positive health outcomes such as lower rates of stunting, mortality and cancer incidences (Jayawardena et al. 2013, Thompson and Meerman 2014).

In addition, information on the underlying contributions of socio-economic factors of household food insecurity, child dietary diversity and child nutritional status is necessary to provide guidance in the management of household food insecurity in a low resource setting, particularly during economic crises. Nigeria experienced two consecutive economic contractions with negative GDP 
growth (-0.36\%) in Q1 (first quarter) 2016, and a larger contraction (-2.06\%) in Q2 (second quarter) 2016 (Teriba 2017) after which by the third quarter of 2016, the Nigerian economy was officially in recession (CBN 2017).

To this effect, the aim of this study is to assess the relationship between household food insecurity and child dietary diversity, as well as identifying coping strategies that households employ to mitigate food insecurity in the households where mother-child pairs live, in an urban population in Ibadan, Nigeria.

\section{Materials and Methods}

\section{Study design and setting}

This is a cross-sectional study conducted between May and June 2017 in three wards of the Ibadan North Local Government in Ibadan, Oyo State. Ibadan is the capital and largest city in Oyo state. It is located in South-Western Nigeria. It is reputed to be the largest indigenous city in Africa south of the Sahara. It is the center of administration of the old Western region. Most of the inhabitants are Yoruba.

\section{Target population and sample size}

The study was conducted among mothers (15 to 49 years) and their children (24 to 59 months) as pairs, in selected households. The sample size was estimated using a two-sided type I error of 5 percent and a statistical power of 80 percent to detect an odds ratio of 1 based on literature (Adebayo and Abegunrin 2013).

\section{Sampling technique and Data collection}

A four-stage sampling procedure was applied in selecting a mother-child pair for the study. In the first stage, Ibadan North local government area (LGA) was purposively selected from a sampling frame of five LGA in the Ibadan metropolis. A sampling frame of the wards in the selected LGA was prepared, and 3 wards were randomly selected from a total of 12 wards. In the selected ward, the total number of households $(N)$ was estimated, and a sampling interval $(i)$ was estimated as the total all households $(N)$ divided by the number of households to be recruited from the ward $(n)$, i.e., $i=N / n$, an index mother to child pair. The index household was selected randomly and every ith household was identified. In each identified household, every eligible mother to child pair who voluntarily gave written (and signed/thumbprint) informed consent to participate in the study was interviewed. Where there was more than one eligible mother to child pair in a household, an index pair was randomly selected. A total of 360 mother-child pairs were interviewed, with information collected from them in a secluded but relaxed atmosphere by trained interviewers.

\section{Research Instruments and Scoring Systems}

The mother or caregiver was requested to provide socio-demographic information, economic characteristics, HFI and CS of households and CDD of the child using 24-hour dietary recall. In addition, anthropometric measurements of the child were taken. 


\section{Household food insecurity (HFI)}

Household food insecurity was measured using the Household Food Insecurity Access Scale (HFIAS) adopted from HFIAS for Measurement of Food Access (Coates, Swindale, and Bilinsky 2007). Mothers were asked nine questions related to the household's experience of food insecurity in the past 30 days. The three domains of food insecurity (1) anxiety and uncertainty about access (1 question), (2) insufficient quality (3 questions), and (3) insufficient quantity (5 questions) were captured in the nine questions. The respondent was first asked an occurrence question - that is, whether the condition in the question happened at all in the past four weeks (yes or no). If the respondent answers "yes" to an occurrence question, a frequency-of-occurrence question is asked to determine whether the condition happened rarely (once or twice), sometimes (three to ten times) or often (more than ten times) and an HFI status was then generated.

\section{Coping strategy (CS)}

A food-related coping strategy is known as one of the indirect methods for assessing adaptive strategies adopted by a household to mitigate the risk of food insecurity. The coping strategy questions include: (1) Do you rely on less preferred food? (2) Do you rely on less expensive foods? (3) Do you borrow money to buy foodstuff? (4) Do you borrow foodstuff? (5) Do you purchase food on credit? (6) Do you rely on help from a relative? (7) Do you limit portions at mealtimes? (8) Do you ration money to the household? (9) Do you limit your own intake? (10) Do you reduce the number of meals? (11) Do you skip the whole day without eating? Details of the design and principles of CS instrument has been reported elsewhere (Maziya-Dixon 2004).

\section{4-hour dietary recall}

The dietary recall was collected for each child using the 24-hour dietary recall multi-pass method. Mothers were asked to recall all edibles (food and drinks) consumed either during the day or at night by the child in the past 24hours. This was used to assess the child's dietary diversity using the Child Dietary Diversity Score (CDDS) adopted from (Ballard 2012). The IYCF minimum dietary diversity is defined as the consumption of 4 or more food groups, meaning the child has a higher likelihood of meeting minimum requirements (Ballard 2012). Seven foods groups were used: (1) Grains, roots and tubers (2) Legumes and nuts (3) Dairy products (milk, yogurt, cheese) (4) Flesh foods (meat, fish, poultry and liver/organ meats) (5) Eggs (6) Vitamin-a rich fruits and vegetables (7) Other fruits and vegetables. Children who consumed $<4$ food groups had low dietary diversity while children who had $\geq 4$ food groups were considered as having high dietary diversity. Details of the methods of data collection and scoring guide are reported in (Ballard 2012).

\section{Anthropometric measurements}

The height and weight of the children were measured using a graduated stadiometer (in $\mathrm{cm}$ ) and weighing scale (in grams) respectively and weight for age (WAZ), height for age (HAZ) and weight for height (WHZ) were compared with reference standards using WHO Anthro Software (V3.1.0). The children were classified into underweight, stunted or wasted if their WAZ, HAZ and WHZ scores were below -2 standard deviations (SD) of the WHO standards.

\section{Data management techniques and statistical analyses}

Responses were coded, and data entered and double-checked to ensure completeness and consistency. Categorical data were presented using frequency (and percentages), but continuous 
data were summarized using mean \pm standard deviation (SD). The Chi-square test was used to assess the relationships between socio-demographic variables and HFI or level of underweight or level of stunting or level of wasting of children. Multivariable logistic regression analysis was applied to estimate the adjusted odds ratio (AOR) and 95\% confidence interval (CI) for the association between HFI status and CDDS (adjusting for socio-demographic factors). Statistical significance of two-sided P $<0.05$ was computed using SPSS for Windows (version 20).

\section{Ethical Considerations and Approval}

The Institution Review Board of the University of Ibadan/University College Hospital (UI/UCH) ethics committee approved the study (UI/EC/17/0219). All respondents were free to rescind from the study at any time without any consequence(s). Confidentiality was effectively addressed by administrating the questionnaire privately, and all identifiers were removed before the data were analyzed.

\section{Results}

\section{Household characteristics of respondents}

The characteristics of respondents and their bivariate associations with HFI and CDDS are present in Table 1. Mother's age was significantly associated with HFI. The proportion of mothers less than or 35 years from food-insecure households $(88.1 \%)$ was significantly $(\mathrm{P}<0.05)$ lower than their counterparts older than 35 years from similar households (96.7\%). Mothers with lower parity had children with higher dietary diversity (73.0\%) than mothers with higher parity (69.1\%) $(\mathrm{P}<0.05)$. CDDS was not related to any of the socio-demographic factors.

\section{HFI, and CS of households in the study}

As illustrated in Figure 1A, 11.4\% of households were severely food insecure, 54.7\% were moderately and $24.2 \%$ mildly food insecure. $81.9 \%$ relied on less expensive foods, $78.3 \%$ rationed money to households, 68.6\% relied on less preferred foods and 69.2\% limited their intake. 54.7\% reduced their meal portion sizes and $48.2 \%$ reduced their daily intakes and $4.2 \%$ went a whole day without eating (Figure 1B).

\section{Anthropometric characteristics and CDDS of children in the study}

In Figure 1C, $26.1 \%$ of the children were stunted, $18.3 \%$ were underweight and $15.6 \%$ were wasted. Among the children, (Figure 1D), 70.0\% had a high dietary diversity score. 
Table 1: Demographic characteristics, HFI and CDDS in the study

\begin{tabular}{|c|c|c|c|c|c|c|c|c|}
\hline \multirow[t]{2}{*}{ Characteristics } & & \multirow{2}{*}{$\begin{array}{c}\text { All } \\
\text { N (\%) }\end{array}$} & \multicolumn{3}{|c|}{ HFI (\%) } & \multicolumn{3}{|c|}{ CDDS (\%) } \\
\hline & & & Food Secure & Food Insecure & P-value & Low & High & P-value \\
\hline \multirow[t]{2}{*}{ Mothers age (years) } & $\leq 35$ & $270(75.0)$ & $32(11.9)$ & $238(88.1)$ & 0.018 & $81(30.0)$ & $189(70.0)$ & 1.000 \\
\hline & $>35$ & $90(25.0)$ & 03 (3.3) & 87 (96.7) & & $27(30.0)$ & $63(70.0)$ & \\
\hline \multirow[t]{3}{*}{ Childs age (months) } & & $42.7 \pm 11.7$ & & & & & & \\
\hline & $<42$ & $166(46.1)$ & $17(10.2)$ & 149 (89.8) & 0.759 & 48 (28.9) & $118(71.1)$ & 0.678 \\
\hline & $\geq 42$ & 194 (53.9) & $18(9.3)$ & $176(90.7)$ & & 60 (30.9) & $134(69.1)$ & \\
\hline \multirow[t]{2}{*}{ Childs sex } & Male & $190(52.8)$ & $19(10.0)$ & $171(90.0)$ & 0.851 & $54(28.4)$ & $136(71.6)$ & 0.489 \\
\hline & Female & $170(47.2)$ & $16(9.4)$ & 154 (90.6) & & $54(31.8)$ & $116(68.2)$ & \\
\hline \multirow[t]{2}{*}{ Parity } & $\leq 3$ & $263(73.1)$ & $28(10.6)$ & 235 (89.4) & 0.330 & $71(27.0)$ & $192(73.0)$ & 0.041 \\
\hline & $>4$ & 97 (26.9) & 07 (7.2) & $90(92.8)$ & & 37 (38.1) & 60 (61.9) & \\
\hline \multirow[t]{2}{*}{ Religion } & Christianity & $224(62.2)$ & $17(7.6)$ & 207 (92.4) & 0.080 & $60(26.8)$ & $164(73.2)$ & 0.088 \\
\hline & Islam & $136(37.8)$ & $18(13.2)$ & 118 (86.8) & & 48 (35.3) & $88(64.7)$ & \\
\hline \multirow[t]{4}{*}{ Tribe } & Yoruba & $291(80.8)$ & 33 (11.3) & 258 (88.7) & 0.118 & 86 (29.6) & $205(70.4)$ & 0.473 \\
\hline & Igbo & $31(8.6)$ & $01(3.2)$ & $30(96.8)$ & & 12 (38.7) & $19(61.3)$ & \\
\hline & Hausa & 07 (1.9) & $01(14.3)$ & $06(85.7)$ & & 03 (42.9) & $04(57.1)$ & \\
\hline & Others & $31(8.6)$ & $0(0.0)$ & $31(100.0)$ & & 07 (22.6) & $24(77.4)$ & \\
\hline \multirow[t]{5}{*}{ Marital status } & Single & $10(2.8)$ & $0(0.0)$ & $10(100.0)$ & 0.772 & $03(30.0)$ & $07(70.0)$ & 0.524 \\
\hline & Married & 344 (95.6) & $35(10.2)$ & 309 (89.8) & & $103(29.9)$ & $241(70.1)$ & \\
\hline & Divorced & $02(0.6)$ & $0(0.0)$ & $02(100.0)$ & & $0(0.0)$ & $02(100.0)$ & \\
\hline & Separated & $01(0.3)$ & $0(0.0)$ & $01(100.0)$ & & $01(100.0)$ & $0(0.0)$ & \\
\hline & Widowed & $03(0.8)$ & $0(0.0)$ & $03(100.0)$ & & 01 (33.3) & 02 (66.7) & \\
\hline \multirow[t]{2}{*}{ Living with spouse } & No & $41(11.4)$ & $0(0.0)$ & $41(100.0)$ & 0.026 & 13 (31.7) & 28 (68.3) & 0.800 \\
\hline & Yes & $319(88.6)$ & $35(11.0)$ & $284(89.0)$ & & 95 (29.8) & $224(70.2)$ & \\
\hline \multirow[t]{2}{*}{ Family type } & Monogamous & 339 (94.2) & $34(10.0)$ & 305 (90.0) & 0.429 & $102(30.1)$ & 237 (69.9) & 0.883 \\
\hline & Polygamous & $21(5.8)$ & $01(4.8)$ & $20(95.2)$ & & $06(28.6)$ & $15(71.4)$ & \\
\hline \multirow[t]{2}{*}{ Household size } & $<5$ & $130(36.1)$ & $13(13)$ & $117(90.0)$ & 0.894 & $34(26.2)$ & 96 (73.8) & 0.231 \\
\hline & $>5$ & $230(63.9)$ & $22(9.6)$ & $208(90.4)$ & & $74(32.2)$ & $156(67.8)$ & \\
\hline Mother's education & $\leq$ Secondary & $255(70.8)$ & $28(11.0)$ & $227(89.0)$ & 0.209 & $82(32.2)$ & $173(67.8)$ & 0.164 \\
\hline
\end{tabular}




\begin{tabular}{|c|c|c|c|c|c|c|c|c|}
\hline \multirow{3}{*}{ Father's education } & Tertiary & 105 (29.2) & $07(6.7)$ & $98(93.3)$ & \multirow{3}{*}{0.785} & $26(24.8)$ & $79(75.2)$ & \multirow{3}{*}{0.102} \\
\hline & $\leq$ secondary & $193(53.6)$ & $18(9.3)$ & 175 (90.7) & & $65(33.7)$ & $128(66.3)$ & \\
\hline & Tertiary & $167(46.4)$ & $17(10.2)$ & $150(89.8)$ & & $43(25.7)$ & 124 (74.3) & \\
\hline Mother's employment & Unemployed & $24(06.7)$ & $01(4.2)$ & $23(95.8)$ & 0.342 & $08(33.3)$ & $16(66.7)$ & 0.712 \\
\hline \multirow{3}{*}{ Father's employment } & Employed & $336(93.3)$ & $34(10.1)$ & $302(89.9)$ & & $100(29.8)$ & $236(70.2)$ & \\
\hline & Unemployed & $05(01.4)$ & $0(0.0)$ & $05(100.0)$ & \multirow[t]{2}{*}{0.460} & $02(40.0)$ & $03(60.0)$ & \multirow[t]{2}{*}{0.623} \\
\hline & Employed & 355 (98.6) & 35 (9.9) & $320(90.1)$ & & 106 (29.9) & $249(70.1)$ & \\
\hline \multirow{2}{*}{$\begin{array}{l}\text { Household monthly } \\
\text { income }\end{array}$} & $\leq \$ 80^{\Phi}$ & $198(55.0)$ & $20(10.1)$ & 178 (89.9) & \multirow[t]{2}{*}{0.789} & $65(32.8)$ & $133(67.2)$ & \multirow[t]{2}{*}{0.195} \\
\hline & $>\$ 80^{\mathrm{T}}$ & $162(45.0)$ & $15(9.3)$ & $147(90.7)$ & & $43(26.5)$ & 119 (73.5) & \\
\hline \multirow[t]{2}{*}{ Food cost* } & $\leq \$ 40^{\Phi}$ & 248 (68.9) & $21(8.5)$ & $227(91.5)$ & \multirow[t]{2}{*}{0.232} & $80(32.3)$ & $168(67.7)$ & \multirow[t]{2}{*}{0.164} \\
\hline & $>\$ 40^{\top}$ & 112 (31.1) & $14(12.5)$ & $98(87.5)$ & & $28(25.0)$ & $84(75.0)$ & \\
\hline
\end{tabular}

* Estimated amount spent on food monthly ${ }^{\mathbf{T}}$ Dollar equivalent at the time of study 
World Nutrition 2020;11(1):129-144

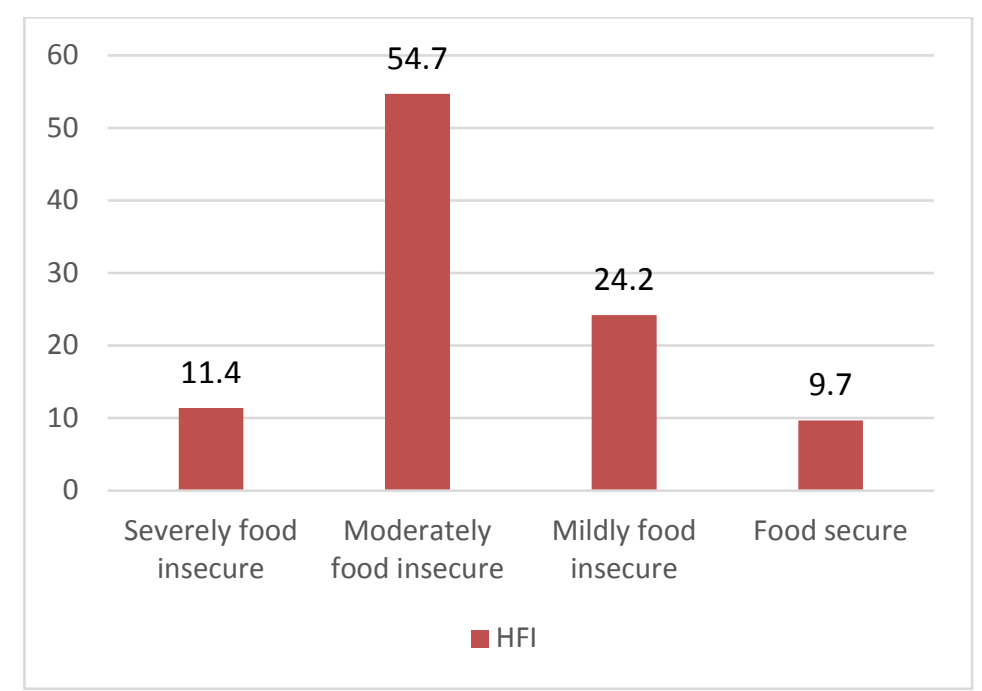

A

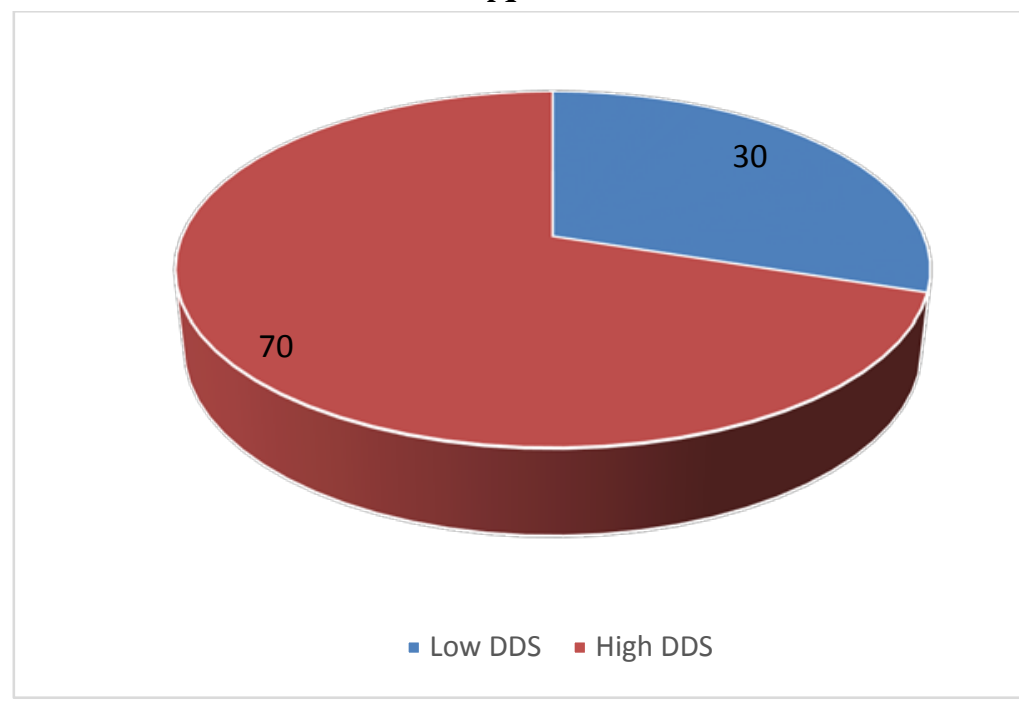

C

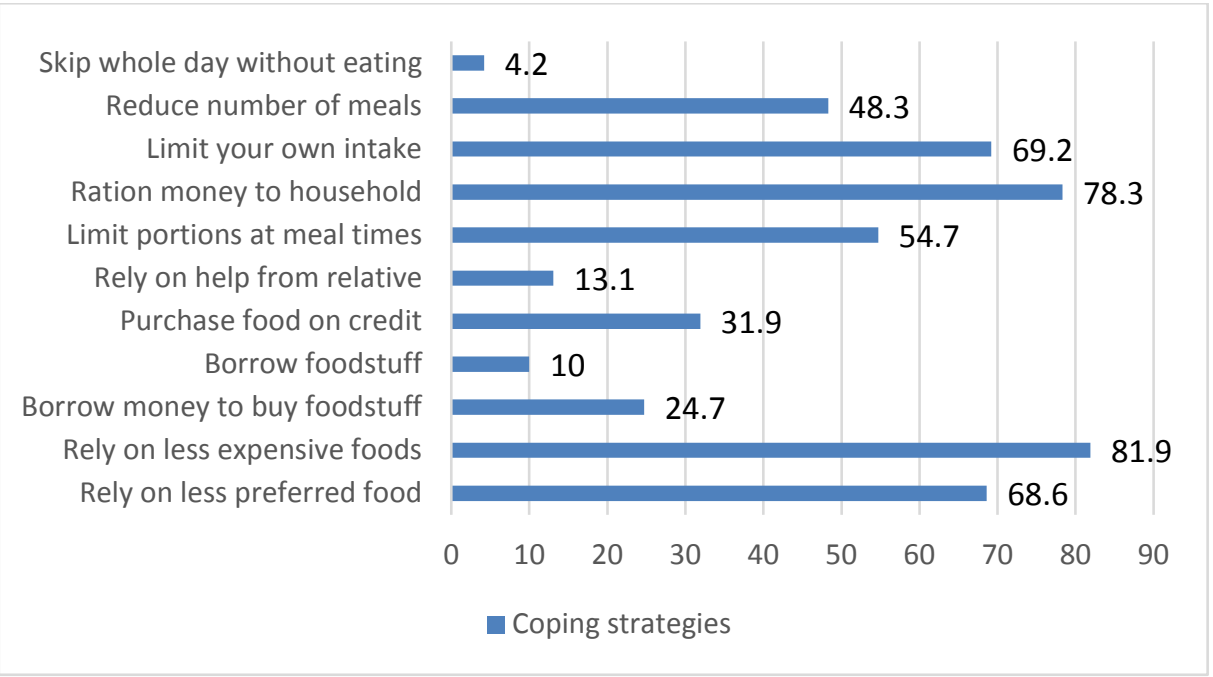

B

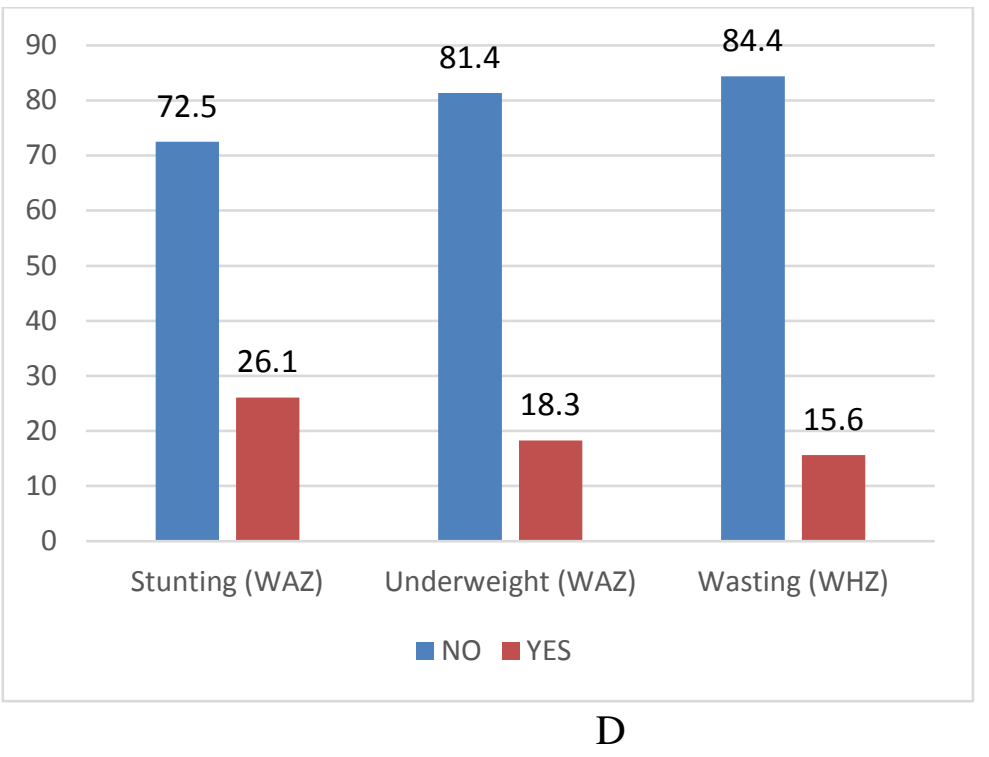

Figure 1: HFI (A) and coping strategies (B) of households in addition to DDS (D) and anthropometric features (C) of children in the study. 
World Nutrition 2020;11(1):129-144

Household characteristics, and nutritional status of children

Mothers' educational status was significantly associated with the child's nutritional status (Table 2). Mothers with at least a tertiary education had a significantly $(\mathrm{P}<0.0001)$ lower proportion of stunted children $(13.6 \%)$ than those with lower educational status $(31.7 \%)$. Similarly, $21.5 \%$ of fathers with at least a tertiary education had a lower proportion of stunted children (21.5\% compared to 30.7\%). Households with a higher monthly income ( $>$ \$8) had less stunting (21.2\%) and underweight (12.3\%) than households with less income.

Table 2: Household characteristics and Nutritional status of children

\begin{tabular}{|c|c|c|c|c|c|c|c|c|c|c|}
\hline \multirow[t]{2}{*}{ Characteristics } & & \multicolumn{2}{|c|}{ Stunting } & \multirow[b]{2}{*}{ P-value } & \multicolumn{3}{|c|}{ Underweight } & \multicolumn{2}{|c|}{ Wasting } & \multirow[b]{2}{*}{ P-value } \\
\hline & & No & Yes & & No & Yes & P-value & No & Yes & \\
\hline \multirow[t]{2}{*}{ Mothers age (years) } & $\leq 35$ & 194 (72.9) & $72(27.1)$ & 0.664 & $221(82.2)$ & $48(17.8)$ & 0.648 & $227(84.1)$ & 43 (15.9) & 0.737 \\
\hline & $>35$ & 67 (75.3) & $22(24.7)$ & & $72(80.0)$ & $18(20.0)$ & & 77 (85.6) & $13(14.4)$ & \\
\hline \multirow[t]{2}{*}{ Childs age (months) } & $<42$ & $119(72.6)$ & $45(27.4)$ & 0.704 & 130 (78.8) & $35(21.1)$ & 0.202 & $137(82.5)$ & $29(17.5)$ & 0.354 \\
\hline & $\geq 42$ & 142 (74.3) & $49(25.7)$ & & $163(84.0)$ & $31(16.0)$ & & 167 (86.1) & 27 (13.9) & \\
\hline \multirow[t]{2}{*}{ Childs sex } & Male & 140 (75.3) & 46 (24.7) & 0.434 & $153(81.0)$ & $36(19.0)$ & 0.732 & 161 (84.7) & $29(15.3)$ & 0.871 \\
\hline & Female & $121(71.6)$ & $48(28.4)$ & & $140(82.4)$ & $30(17.6)$ & & $143(84.1)$ & $27(15.9)$ & \\
\hline \multirow[t]{2}{*}{ Parity } & $\leq 3$ & 194 (74.9) & $65(25.1)$ & 0.332 & $215(82.1)$ & 47 (17.9) & 0.720 & $221(84.0)$ & $42(16.0)$ & 0.721 \\
\hline & $>4$ & 67 (69.8) & $29(30.2)$ & & $78(80.4)$ & $19(19.6)$ & & $83(85.6)$ & $14(14.4)$ & \\
\hline \multirow[t]{2}{*}{ Religion } & Christianity & 165 (75.3) & $54(24.7)$ & 0.324 & 180 (80.7) & 43 (19.3) & 0.574 & 183 (81.7) & 41 (18.3) & 0.065 \\
\hline & Islam & $96(70.6)$ & $40(29.4)$ & & 113 (83.1) & 23 (16.9) & & $121(89.0)$ & $15(11.0)$ & \\
\hline \multirow[t]{4}{*}{ Tribe } & Yoruba & 215 (74.7) & $73(25.3)$ & 0.772 & 237 (81.7) & $53(18.3)$ & 0.580 & 244 (83.8) & $47(16.2)$ & 0.471 \\
\hline & Igbo & 20 (66.7) & 10 (33.3) & & $25(80.6)$ & 06 (19.4) & & $25(80.6)$ & 06 (19.4) & \\
\hline & Hausa & 05 (71.4) & $02(28.6)$ & & 07 (100.0) & $0(0.0)$ & & 07(100.0) & $0(0)$ & \\
\hline & Others & $21(70.0)$ & $09(30.0)$ & & $24(77.4)$ & 07 (22.6) & & 28 (90.3) & $03(9.7)$ & \\
\hline \multirow[t]{5}{*}{ Marital status } & Single & $06(60.0)$ & $04(40.0)$ & 0.457 & $10(100.0)$ & $0(0.0)$ & 0.364 & 09 (90.0) & $01(10.0)$ & 0.159 \\
\hline & Married & 251 (73.8) & 89 (26.2) & & 279 (81.1) & 65 (18.9) & & $290(84.3)$ & 54 (15.7) & \\
\hline & Divorced & $01(50.0)$ & $01(50.0)$ & & $01(50.0)$ & $01(50.0)$ & & $02(100.0)$ & $0(0.0)$ & \\
\hline & Separated & $0(0.0)$ & $0(0.0)$ & & 01 (100.0) & $0(0.0)$ & & $0(0.0)$ & $01(100.0)$ & \\
\hline & Widowed & 03 (100.0) & $0(0.0)$ & & $02(100.0)$ & $0(0.0)$ & & 03 (100.0) & $0(0.0)$ & \\
\hline \multirow[t]{2}{*}{ Living with spouse } & No & 31 (79.5) & $08(20.5)$ & 0.371 & $34(85.0)$ & $06(15.0)$ & 0.558 & $36(87.8)$ & $05(12.2)$ & 0.528 \\
\hline & Yes & 230 (72.8) & $86(27.2)$ & & 259 (81.2) & 60 (18.8) & & 268 (84.0) & $51(16.0)$ & \\
\hline Family type & Monogamous & 243 (72.8) & $91(27.2)$ & 0.192 & 273 (80.8) & $65(19.2)$ & 0.097 & 285 (84.1) & 54 (15.9) & 0.432 \\
\hline
\end{tabular}




\begin{tabular}{|c|c|c|c|c|c|c|c|c|c|c|}
\hline World Ne & $\begin{array}{l}\text { ition 2020;11 } \\
\text { Polygamous }\end{array}$ & $\begin{array}{l}129-144 \\
18(85.7)\end{array}$ & 03 (14.3) & & 20 (95.2) & $01(4.8)$ & & 19 (90.5) & 02 (9.5) & \\
\hline \multirow[t]{2}{*}{ Household size } & $<5$ & $89(69.5)$ & 39 (30.5) & 0.201 & $104(80.6)$ & $25(19.4)$ & 0.715 & 109 (83.8) & $21(16.2)$ & 0.814 \\
\hline & $>5$ & $172(75.8)$ & $55(24.2)$ & & $189(82.2)$ & $41(17.8)$ & & $195(84.8)$ & $35(15.2)$ & \\
\hline \multirow[t]{2}{*}{ Mother's education } & $\leq$ Secondary & $172(68.3)$ & $80(31.7)$ & 0.000 & $202(79.2)$ & $53(20.8)$ & 0.066 & 215 (84.3) & $40(15.7)$ & 0.915 \\
\hline & Tertiary & $89(86.4)$ & $14(13.6)$ & & $91(87.5)$ & $13(12.5)$ & & $89(84.8)$ & $16(15.2)$ & \\
\hline \multirow[t]{2}{*}{ Father's education } & $\leq$ secondary & $133(69.3)$ & $59(30.7)$ & 0.049 & $154(79.8)$ & $39(20.2)$ & 0.336 & $161(83.4)$ & $32(16.6)$ & 0.564 \\
\hline & Tertiary & 128 (78.5) & 35 (21.5) & & 139 (83.7) & 27 (16.3) & & $143(85.6)$ & $24(14.4)$ & \\
\hline \multirow[t]{2}{*}{ Mother's employment } & Unemployed & $20(87.0)$ & $03(13.0)$ & 0.131 & $22(91.7)$ & $02(8.3)$ & 0.188 & $22(91.7)$ & $02(8.3)$ & 0.312 \\
\hline & Employed & $241(72.6)$ & $91(27.4)$ & & $271(80.9)$ & 64 (19.1) & & $282(83.9)$ & $54(16.1)$ & \\
\hline \multirow[t]{2}{*}{ Father's employment } & Unemployed & $02(40.0)$ & $03(60.0)$ & 0.087 & $03(60.0)$ & $02(40.0)$ & 0.209 & $05(100.0)$ & $0(0.0)$ & 0.334 \\
\hline & Employed & $259(74.0)$ & $91(26.0)$ & & $290(81.9)$ & $64(18.1)$ & & $299(84.2)$ & $56(15.8)$ & \\
\hline \multirow{2}{*}{$\begin{array}{l}\text { Household monthly } \\
\text { Income }\end{array}$} & $\leq \$ 80^{\text {T }}$ & 135 (69.2) & $60(30.8)$ & 0.043 & $151(76.6)$ & $46(23.4)$ & 0.007 & $161(81.3)$ & 37 (18.7) & 0.070 \\
\hline & $>\$ 80^{\mp}$ & 126 (78.8) & $34(21.2)$ & & 142 (87.7) & 20 (12.3) & & 143 (88.3) & 19 (11.7) & \\
\hline \multirow[t]{2}{*}{ Food cost* } & $\leq \$ 40^{\mp}$ & $176(71.8)$ & $69(28.2)$ & 0.283 & $198(80.2)$ & 49 (19.8) & 0.291 & $211(85.1)$ & 37 (14.9) & 0.620 \\
\hline & $>\$ 40^{\mp}$ & 85 (77.3) & $25(22.7)$ & & 95 (84.8) & $17(15.2)$ & & $93(83.0)$ & $19(17.0)$ & \\
\hline
\end{tabular}

* Estimated amount spent on food monthly $\quad{ }^{\mathrm{\Phi}}$ Dollar equivalent at the time of study

\section{HFI, CDDS and nutritional status of children in the study}

As shown in Table 3, food-insecure households were likely $(\mathrm{P}<0.05)$ to have children with low dietary diversity (32.0\%) compared to food-secure households (11.4\%). HFI was statistically insignificantly associated with the nutritional status of the children.

Table 3: Household food insecurity, CDDS and nutritional status of children

\begin{tabular}{|c|c|c|c|c|c|c|c|c|c|c|c|c|c|}
\hline & & \multicolumn{3}{|c|}{ CDDS } & \multicolumn{3}{|c|}{ Stunting } & \multicolumn{3}{|c|}{ Underweight } & \multicolumn{3}{|c|}{ Wasting } \\
\hline & & High & Low & $P$-value & No & Yes & $P$-value & No & Yes & P-value & No & Yes & P-value \\
\hline HFI & $\begin{array}{l}\text { Food secure } \\
\text { Food insecure }\end{array}$ & $\begin{array}{l}31(88.6) \\
221(68.0)\end{array}$ & $\begin{array}{l}4(11.4) \\
104(32.0)\end{array}$ & 0.012 & $\begin{array}{l}28(82.4) \\
233(72.6)\end{array}$ & $\begin{array}{l}6(17.6) \\
88(27.4)\end{array}$ & 0.220 & $\begin{array}{l}28(80.0) \\
265(81.8)\end{array}$ & $\begin{array}{l}7(20.0) \\
59(18.2)\end{array}$ & 0.795 & $\begin{array}{l}27(77.1) \\
277(85.2)\end{array}$ & $\begin{array}{l}8(22.9) \\
48(14.8)\end{array}$ & 0.210 \\
\hline
\end{tabular}




\section{Adjusted odds of CDDS}

Adjusted odds of high CDDS given HFI (Table 4) was 0.266, 95\% confidence interval (0.091, 0.783), P = 0,016. This implies that food-insecure households are about four times less likely to have children with high CDDS.

\begin{tabular}{lcc}
\multicolumn{2}{l}{ Table 4. Independent association of socio-demographic factors and HFI status with high CDDS } \\
\hline Factors & Adjusted OR (95\%CI) & P-value \\
\hline Mothers age (>35 years) & $1.231(0.713,2.126)$ & 0.456 \\
Parity $(>4)$ & $0.619(0.369,1.039)$ & 0.070 \\
Mothers education (Tertiary education) & $1.165(0.610,2.225)$ & 0.644 \\
Fathers education (Tertiary education) & $1.261(0.714,2.229)$ & 0.424 \\
Household monthly income $(>\$ 80)$ & $1.2140 .747,1.973)$ & 0.434 \\
HFI (Food insecure) & $0.266(0.091,0.783)$ & 0.016 \\
\hline
\end{tabular}

\section{DISCUSSION}

This study provides insights into the relationship between HFI and CDD in a sample of urban households in Ibadan, Nigeria. We found HFI was inversely associated with CDD. Our sample had a higher prevalence of HFI (90.3\%) than has been reported in other studies (69.2\% in Nepal (Osei et al. 2010), 65\% (Ajao et al. 2010) and 70.0\% (Adebayo and Abegunrin 2013) in Nigeria) but similar to the study in Uganda (Whitney et al. 2018). This infers that food insecurity is a situation of concern among the study population. The present study was conducted at a time the Nigerian economy suffered a recession, a contributing cause of food insecurity, as it results in a decline in GDP, household income, etc. (CBN 2012). Nigeria was recorded to have experienced deterioration in food security from 2016 to 2018 (FAO 2018).

In response to HFI, households resorted to a number of coping strategies, with the consumption of less expensive foods being the most frequently used strategy which is consistent with findings from Ethiopia, Lao PDR, and Nigeria (Adebayo and Abegunrin 2013, Asesefa Kisi et al. 2018, Broz 2014). This is most likely due to the inflation of prices of food during the period of data collection. However, other studies in South Africa, Malaysia, Nigeria and Ethiopia (Oldewage-Theron, Dicks, and Napier 2006, Shariff and Khor 2008, Ibrahim et al. 2009, Birhane et al. 2014) found limiting portion sizes, reducing meal intake outside the home, purchase of less preferred foods as the most frequently used coping mechanism by the respondents. Other coping strategies used by our sample women are shown in Figure 1B. Other studies have documented the following strategies: borrowing food or money to buy food, selling productive assets like livestock, reducing quantity and frequency of meal, purchase food on credit, getting help from relatives, working in exchange for food and selling of assets in exchange of food, eating less (and less nutritious) meals, sticking to a budget, maintain a food garden, send household members to eat elsewhere, send households members to beg, gather wild food, buying necessities only, eating less preferred meals, mothers giving up their meals for the children, skipping meals, going a whole day without food (FAO 2015, Asesefa Kisi et al. 2018, Oldewage-Theron, Dicks, and Napier 2006, Broz 2014, Kruger, Schönfeldt, and Owen 2008, Maxwell 1996, McCarthy, Chang, and Brimblecombe 2018, Tsegaye et al. 2018).

A relationship was found between household food insecurity and dietary diversity of children, and when adjusted for odds, food-insecure households were about 4 times less likely to have children with high dietary diversity, similar to the results obtained among children on San Cristobal Island, Galapagos (Pera, Katz, and Bentley 2019). UNICEF explains that in times of financial crises, children are consciously and unconsciously affected by changes in their diets with a decline in their daily nutritional intake and consumption of nutritious food as a result of a shortfall in family income (UNICEF 2014). Despite the reported food insecurity in our sample, dietary diversity among most children was high. However, a statistically significant relationship was found between food insecure 
households and children with low dietary diversity. (Maitra 2018) explained that the mediating role of dietary diversity in the relationship between food insecurity and nutritional status is mixed and dependent on the type of survey (longitudinal versus cross-sectional), age-group studied, and the location (LMIC or high-income countries).

Regarding malnutrition, which has remained a public health challenge in Nigeria, the percentage of children stunted, wasted and underweight in this study was similar to data 2018 Nigeria Demographic and Health Survey (National Population Commission 2019). The nutritional status of children through compromised quantity and quality of dietary intake is undeniably affected by household food insecurity (Ali et al. 2013) in situations of poor food availability, access, utilization and stability of supply. Although in this present study, no significant association was found between HFI and child nutritional status, this is in contrast to other studies (Abdurahman et al. 2016, Ali et al. 2013, Singh, Singh, and Ram 2014) but similar to a study in Nepal (Osei et al. 2010). This may be due to the categorization of food insecurity into two categories, as having four categories is more likely to provide a robust association with malnutrition among children than with only two variables (Singh, Singh, and Ram 2014). That food-secure households were found to have children who had more diverse diets is similar to studies from other regions (Ali et al. 2013, Aurino and Morrow 2018, Chandrasekhar and Aguayo 2017).

Socioeconomic status (SES) encompasses more than just income but also includes educational attainment, financial security, and subjective perceptions of social status and social class, quality of life attributes as well as opportunities and privileges afforded and is a key factor in determining the quality of life for women, by extension children and families and household food security (APA 2019, Sanusi, Badejo, and Yusuf 2006). The UNICEF conceptual framework relates limited resources, educational status, etc. to household food access and ultimately child undernutrition (Black et al. 2008). In this study, mothers' educational status was significantly associated with the child's nutritional status, in agreement with other authors (Oyebanjo, Ambali, and Akerele 2013, Roberts, Osadare, and Inem 2019, Singh, Singh, and Ram 2014). This implies that the more educated the father and/or mother, the more food secure the household (Babatunde, Omotesho, and Sholotan 2007). Maternal education has been consistently shown to be critically important for child health, nutrition, and survival. The precise mechanism by which maternal education affects child outcomes is not fully understood but evidence from various countries indicates that knowledge and practices are key pathways. Hence, it can be said that educated women are more likely to be more aware of nutrition, hygiene and health care than uneducated women (Ajao et al. 2010). (Mulu and Mengistie 2017) found that maternal education can improve a child's nutrition by increasing income and control of household resources. Younger mothers with lower parity were also more likely to be from food-secure households.

A limitation of the present study is that the questions in the food security indicator used (HFIAS) addressed the situation of all household members but did not distinguish adults from children. This study also did not take into consideration the household dietary diversity which can also be used to determine the extent of household food insecurity.

\section{CONCLUSIONS}

This study found a very high percentage of the households to be food-insecure and this was associated with the nutritional status of the children, who had high rates of stunting and underweight. It also found an association between household food insecurity and low children's dietary diversity. We postulate that the findings could be a reflection of the present state of economic recession in the country at the time of the study. It will, therefore, be recommended that strategies and interventions should be implemented to cushion the effect of economic recession on food security status. Maternal sensitization on how to maximize the available limited resources as part of household coping interventions is needed to mitigate the consequences among young children in the households. 


\section{REFERENCES}

Abdurahman, A. A., K. Mirzaei, A. R. Dorosty, A. Rahimiforoushani, and H. Kedir. 2016. "Household Food Insecurity May Predict Underweightand Wasting among Children Aged 24-59 Months." Ecol Food Nutr no. 55 (5):456-72. doi: 10.1080/03670244.2016.1207069.

Adebayo, Oyefunke Olayemi, and Oluwasogo Dammy Abegunrin. 2013. "Analysis of Food Insecurity and Coping Mechanisms among Rural Women in Boripe Local Government Area Of Osun State, Nigeria." Journal of International Institute for Science, Technology and Education no. 3 (20):9-16.

Ajao, KO, EO Ojofeitimi, AA Adebayo, AO Fatusi, and OT Afolabi. 2010. "Influence of family size, household food security status, and child care practices on the nutritional status of under-five children in Ile-Ife, Nigeria." African journal of reproductive health no. 14 (4).

Ali, Disha, Kuntal K. Saha, Phuong H. Nguyen, Michael T. Diressie, Marie T. Ruel, Purnima Menon, and Rahul Rawat. 2013. "Household food insecurity is associated with higher child undernutrition in Bangladesh, Ethiopia, and Vietnam, but the effect is not mediated by child dietary diversity." The Journal of nutrition no. 143 (12):2015-2021. doi: 10.3945/jn.113.175182.

APA. 2019. American Psychological Association. Socioeconomic status office. Resources and publications.

Asesefa Kisi, Misgana, Dessalegn Tamiru, Melese Sinaga Teshome, Meseret Tamiru, and Garumma Tolu Feyissa. 2018. "Household food insecurity and coping strategies among pensioners in Jimma Town, South West Ethiopia." BMC Public Health no. 18 (1):1373. doi: 10.1186/s12889-018-6291-y.

Aurino, Elisabetta, and Virginia Morrow. 2018. "“Food prices were high, and the dal became watery". Mixed-method evidence on household food insecurity and children's diets in India." World Development no. 111:211-224. doi: https://doi.org/10.1016/j.worlddev.2018.07.009.

Babatunde, RO, OA Omotesho, and OS Sholotan. 2007. "Socio-economic characteristics and food security status of farming households in Kwara State, North-Central Nigeria." Pakistan Journal of Nutrition no. 6 (1):49-58.

Ballard, Terri. 2012. "Measuring dietary diversity: beyond households and scores." world Bank Workshop on Food Security and Nutrition: From Measurement to Results.

Birhane, T., S. Shiferaw, S. Hagos, and K. S. Mohindra. 2014. "Urban food insecurity in the context of high food prices: a community based cross sectional study in Addis Ababa, Ethiopia." BMC Public Health no. 14:680. doi: 10.1186/14712458-14-680.

Black, Robert E., Lindsay H. Allen, Zulfiqar A. Bhutta, Laura E. Caulfield, Mercedes de Onis, Majid Ezzati, Colin Mathers, and Juan Rivera. 2008. "Maternal and child undernutrition: global and regional exposures and health consequences." The Lancet no. 371 (9608):243-260. doi: https://doi.org/10.1016/S0140-6736(07)61690-0.

Black, Robert E., Cesar G. Victora, Susan P. Walker, Zulfiqar A. Bhutta, Parul Christian, Mercedes de Onis, Majid Ezzati, Sally Grantham-McGregor, Joanne Katz, Reynaldo Martorell, and Ricardo Uauy. 2013. "Maternal and child undernutrition and overweight in low-income and middle-income countries." The Lancet no. 382 (9890):427-451. doi: https://doi.org/10.1016/S0140-6736(13)60937-X.

Broz, Jessica Lynn. 2014. Are Coping Strategies Used by Households During Times of Food Insecurity Associated with Nutritional Status for Children Under 5 in Lao PDR? Doctoral dissertation.

CBN. 2012. "Central Bank of Nigeria. Understanding monetary policy series no 14. Economic recession.".

CBN. 2017. Recession and Exchange Rate Crisis: The Role of Monetary Policy. In Economic And Financial Review.

Chandrasekhar, S., and V. M. Aguayo. 2017. "Household food insecurity and children's dietary diversity and nutrition in India. Evidence from the comprehensive nutrition survey in Maharashtra." no. 13 Suppl 2. doi: 10.1111/mcn.12447.

Coates, Jennifer, Anne Swindale, and Paula Bilinsky. 2007. "Household Food Insecurity Access Scale (HFIAS) for measurement of food access: indicator guide: version 3."

FAO, IFAD, UNICEF, WFP, and WHO. 2018. "The state of food security and nutrition in the world 2018. Building climate resilience for food security and nutrition.".

FAO, IFAD, WFP. 2015. "The State of Food Insecurity in the World 2015. Meeting the 2015 international hunger targets: taking stock of uneven progress." 
Farzana, Fahmida Dil, Ahmed Shafiqur Rahman, Sabiha Sultana, Mohammad Jyoti Raihan, Md Ahshanul Haque, Jillian L. Waid, Nuzhat Choudhury, and Tahmeed Ahmed. 2017. "Coping strategies related to food insecurity at the household level in Bangladesh." PloS one no. 12 (4):e0171411-e0171411. doi: 10.1371/journal.pone.0171411.

Ibrahim, H, NR Uba-Eze, SO Oyewole, and EG Onuk. 2009. "Food security among urban households: a case study of Gwagwalada area council of the federal capital territory Abuja, Nigeria." Pakistan Journal of Nutrition no. 8 (6):810813.

Jayawardena, Ranil, Nuala M Byrne, Mario J Soares, Prasad Katulanda, Bijesh Yadav, and Andrew P Hills. 2013. "High dietary diversity is associated with obesity in Sri Lankan adults: an evaluation of three dietary scores." BMC public health no. $13(1): 314$.

Kennedy, Gina, Terri Ballard, and Marie Claude Dop. 2011. Guidelines for measuring household and individual dietary diversity: Food and Agriculture Organization of the United Nations.

Kruger, Rozanne, Hettie Carina Schönfeldt, and Johanna Hendriena Owen. 2008. "Food-Coping Strategy Index Applied to a Community of Farm-Worker Households in South Africa." Food and Nutrition Bulletin no. 29 (1):3-14. doi: $10.1177 / 156482650802900101$.

Maitra, Chandana. 2018. A review of studies examining the link between food insecurity and malnutrition: Food and Agriculture Organization of the United Nations.

Maxwell, Daniel G. 1996. "Measuring food insecurity: the frequency and severity of "coping strategies". "Food Policy no. 21 (3):291-303. doi: https://doi.org/10.1016/0306-9192(96)00005-X.

Maziya-Dixon, B. 2004. Nigeria food consumption and nutrition survey 2001-2003: summary: IITA.

McCarthy, Leisa, Anne B. Chang, and Julie Brimblecombe. 2018. "Food Security Experiences of Aboriginal and Torres Strait Islander Families with Young Children in An Urban Setting: Influencing Factors and Coping Strategies." International journal of environmental research and public health no. 15 (12):2649. doi: 10.3390/ijerph15122649.

Mulu, Ermiyas, and Bezatu Mengistie. 2017. "Household food insecurity and its association with nutritional status of under five children in Sekela District, Western Ethiopia: a comparative cross-sectional study." BMC Nutrition no. 3 (1):35.

National Population Commission, Nigeria. 2019. "Nigeria Demographic and Health Survey 2018 Key Indicators Report." Abuja, Nigeria, and Rockville, Maryland, USA:NPC and ICF.

Ogundari, Kolawole. 2013. Determinants of food-poverty states and the demand for dietary diversity in Nigeria.

Oldewage-Theron, Wilna H., Emsie G. Dicks, and Carin E. Napier. 2006. "Poverty, household food insecurity and nutrition: Coping strategies in an informal settlement in the Vaal Triangle, South Africa." Public Health no. 120 (9):795-804. doi: https://doi.org/10.1016/j.puhe.2006.02.009.

Osei, Akoto, Pooja Pandey, David Spiro, Jennifer Nielson, Ram Shrestha, Zaman Talukder, Victoria Quinn, and Nancy Haselow. 2010. "Household food insecurity and nutritional status of children aged 6 to 23 months in Kailali District of Nepal." Food and Nutrition Bulletin no. 31 (4):483-494.

Oyebanjo, O, OI Ambali, and EO Akerele. 2013. "Determinants of food security status and incidence of food insecurity among rural farming households in Ijebu Division of Ogun State, Nigeria." Journal of Agricultural Science and Environment, No13:92-103.

Pera, Megan F., Beth N. H. Katz, and Margaret E. Bentley. 2019. "Dietary Diversity, Food Security, and Body Image among Women and Children on San Cristobal Island, Galapagos." Maternal and Child Health Journal no. 23 (6):830-838. doi: 10.1007/s10995-018-02701-4.

Roberts, Alero Ann, James Olufemi Osadare, and Victor Akpan Inem. 2019. "Hunger in the midst of plenty: A survey of household food security among urban families in Lagos State, Nigeria." Journal of public health in Africa no. 10 (1).

Sanusi, Ajani Rasaki, Adebukola Catherine Badejo, and Oyindamola Bidemi Yusuf. 2006. "Measuring household food insecurity in selected local government areas of Lagos and Ibadan, Nigeria." Pakistan journal of Nutrition no. 5 (1):6267.

Shariff, Zalilah Mohd, and Geok Lin Khor. 2008. "Household food insecurity and coping strategies in a poor rural community in Malaysia." Nutrition research and practice no. 2 (1):26-34.

Singh, A., A. Singh, and F. Ram. 2014. "Household food insecurity and nutritional status of children and women in Nepal." Food Nutr Bull no. 35 (1):3-11. doi: 10.1177/156482651403500101. 
Snel, Erik, and Richard Staring. 2001. "Poverty, migration, and coping strategies: an introduction." Focaal European journal of anthropology no. 38:7-22.

Teriba, Ayo. 2017. "Nigeria's Economic Outlook in 2017." Available at SSRN 2939899.

Thompson, Brian, and Janice Meerman. 2014. "19 Towards Long-term Nutrition Security: The Role of Agriculture in Dietary Diversity." Improving Diets and Nutrition:246.

Tsegaye, A. T., A. Tariku, A. G. Worku, S. M. Abebe, M. Yitayal, T. Awoke, K. Alemu, and G. A. Biks. 2018. "Reducing amount and frequency of meal as a major coping strategy for food insecurity." Arch Public Health no. 76:56. doi: 10.1186/s13690-018-0303-3.

UNICEF. 2014. "Children of the Recession: The impact of the economic crisis on child well-being in rich countries." Innocenti Report Card 12, UNICEF Office of Research, Florence.

Whitney, Cory William, Eike Luedeling, Oliver Hensel, John RS Tabuti, Michael Krawinkel, Jens Gebauer, and Katja Kehlenbeck. 2018. "The role of homegardens for food and nutrition security in Uganda." Human Ecology no. 46 (4):497-514. 